\title{
Low Voltage Energy Harvesting by an Efficient AC-DC Step-Up Converter
}

\author{
K. Madhuri , Dr.A Srujana \\ Student in EEE Department Professor \& HOD for EEE Department Sri Venkateshwara Engineering College, \\ Suryapet
}

\begin{abstract}
In this paper, a direct ac-to-dc power converter is proposed for efficient energy harvesting from the low voltage inertial micro generators. The conventional power converters use diode bridge rectifiers and condition the micro generator outputs in two stages. Hence, they are inefficient and may not be a feasible option for very low voltage micro generators. Moreover, they are not conducive for optimum energy harvesting. The proposed converter avoids the use of bridge rectifiers, and directly converts the ac input to the required dc output. This converter uses a boost converter and a buck-boost converter to process the positive and negative half cycles of the ac input voltage, respectively. Furthermore, using this converter, maximum energy harvesting can be implemented effectively. Analysis of the converter is carried out. Based on the analysis, two schemes are proposed to control the converter. Design guidelines are presented for selecting the converter component and control parameters. A self-starting circuit is proposed for independent operation of the converter. Simulation results are presented to validate the proposed converter topology and control scheme.
\end{abstract}

Key words: $A C-D C$ conversion, boost converter, energy harvesting, low power, low voltage, power converter control.

\section{Introduction}

The recent development of compact and efficient semi conductor technologies has enabled the development of low-power wireless devices. Typical applications for such devices are wireless sensor nodes for structural monitoring, data transfer, biomedical implants etc.

Batteries have been traditionally used as the energy source for such low-power wireless applications. However, they are inherently limited by capacity and size considerations. Therefore, they need to be recharged and replaced periodically. For low-power requirement of a few milli watts, harvesting energy from the environment has become feasible option.

Vibration based energy harvesting is a popular way of extracting electrical energy from the environment. In particular, electromagnetic micro generators work on the principle of faraday's law of electromagnetism. They utilize ambient vibrations to enable movement of a permanent magnet which induces an electromotive force in a stationary coil. The amount of harvested energy can be controlled by changing the load resistance connected to the coil. Unlike other popular vibration-based generators like piezo-electric micro generators, electromagnetic generators have to be specifically designed for a particular environment.

Many types of micro generators, used in the self-powered devices, are reported in the literature for harvesting different forms of ambient energies [1]-[6]. The power level of the inertial -micro-generators is normally very low ranging from few microwatts to tens of mill watts. Based on the energy conversion principle, the inertial micro-generators can be classified mainly into three types: electromagnetic, piezoelectric, and electrostatic [5], [6], among them, the electromagnetic micro-generators have the highest energy density [5], [7] $-[10]$.

The electromagnetic generators are typically spring-mass damper- based resonance systems 'as shown in Fig. 1' in which the small amplitude ambient mechanical vibrations are amplified into larger amplitude translational movements and the mechanical energy of the motion is converted to electrical energy by electromagnetic coupling.

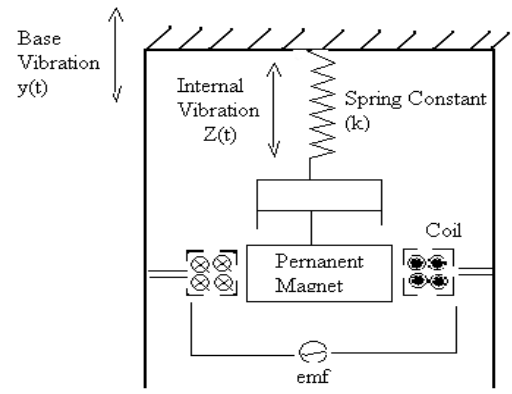

Fig .1 Schematic diagram of an inertial micro generator 
An electromagnetic power generator consists of a copper coil, a permanent magnet (also acting as a mass), and a spring, the permanent magnet is attached to the coil through the spring. This generator works when there is a vibration input, the coil cuts through the magnetic flux formed by the permanent magnet due to the relative displacement between the permanent magnet and the coil. A sinusoidal electromotive force (EMF) in the coil can be generated and thus transfers mechanical energy into electrical energy. Since the output power of the power generator is very low, ranging from few micro-watts to tens of mill-watts, an energy harvesting interface circuit with high power transfer efficiency need to recharge and store the electrical power into the energy storage elements.

In the electromagnetic micro-generators, due to practical size limitations, the output voltage level of the generators is very low (few hundreds of mill volts), whereas the electronic loads require much higher dc voltage $(3.3 \mathrm{~V})$ [9]. The conventional power converters, reported for energy harvesting [3] - [5], mostly Consist of two stages, a diode bridge rectifier and a standard buck or boost dc-to-dc converter. However, there are major disadvantages in using the two-stage power converters to condition the outputs of the electromagnetic microgenerators. For very low-voltage electromagnetic micro-generators, rectification is not feasible by the use of conventional diodes.

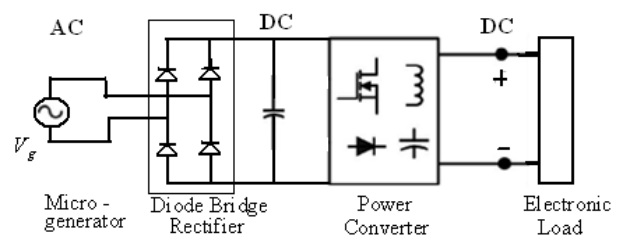

Fig.2conventional two-stage power conversion.

To address the problems of the two-stage power converters a new direct ac-to-dc power converter 'as shown in Fig.3', without conventional diode bridge rectifiers.

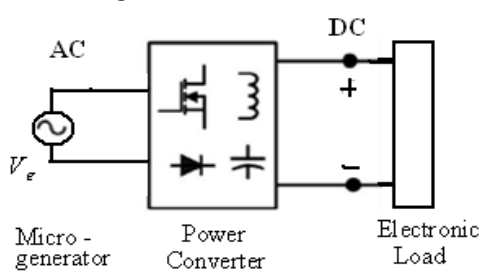

Fig. 3 direct ac-to-dc power conversion

A dual polarity boost converter topology for such ac-dc conversion was proposed 'as shown in Fig.4'.

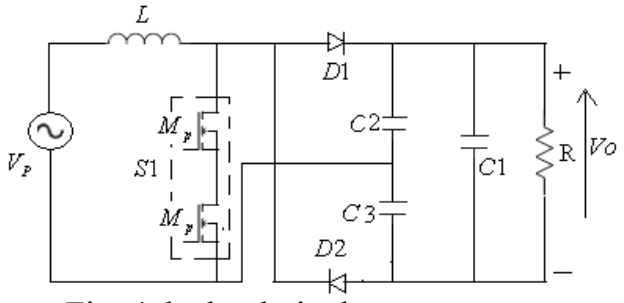

Fig. 4 dual polarity boost converter

This converter uses two inductors and the output dc bus is split into two series connected capacitors. The fundamental principle behind the proposed topology is the use of two boost converters each operating in one half cycle of the ac voltage. Each capacitor is charged only in the respective half cycle. However, they discharge to the load continuously causing large voltage drops. Extremely large capacitors are needed to make the voltage ripple acceptable. This makes the converter response very slow; the control of the converter also requires input line polarity sensing adding further complexity.

Since the output dc bus is split into two series connected capacitors and each of these capacitors is charged only for one half cycle of the micro generator output voltage. As the time periods of the resonancebased micro generators' output voltages are normally in the order of milliseconds, very large voltage drops will occur in the capacitors during the half cycles when they are not charged by the converter. Extremely large capacitors will be required to achieve acceptable voltage ripple at the output dc bus. This is not practical due to the size limitations of the micro generators.

To address the problems of the conventional two stage converters, direct ac-to-dc converter is proposed (11). The proposed converter is shown in fig. 5 .The proposed converter consists of a boost converter in parallel with a buck-boost converter. The output dc bus is realized by using a single capacitor. The output capacitor is charged by the boost converter in the positive half cycle and by the buck-boost converter in the negative half cycle. Therefore, it resolves the problems present in a dual-polarity boost converter. 


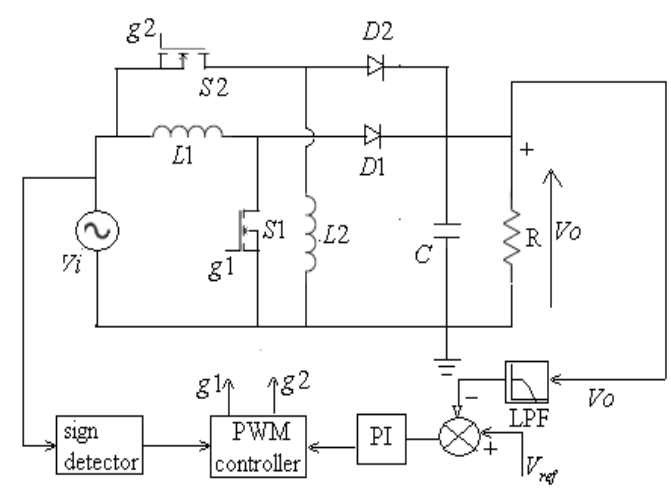

Fig .5 proposed direct ac-to-dc converter

The proposed direct ac-to-dc converter utilizes the negative output to input voltage gain of a buckboost converter to step-up the negative half input voltage of the micro generator to a positive high-dc output voltage.

The H-bridge converter topology for direct ac-to-dc boost conversion(12), standard 4-switch H-bridge converter or the 2-switch,' as shown in Fig.6(a), and (b)'.
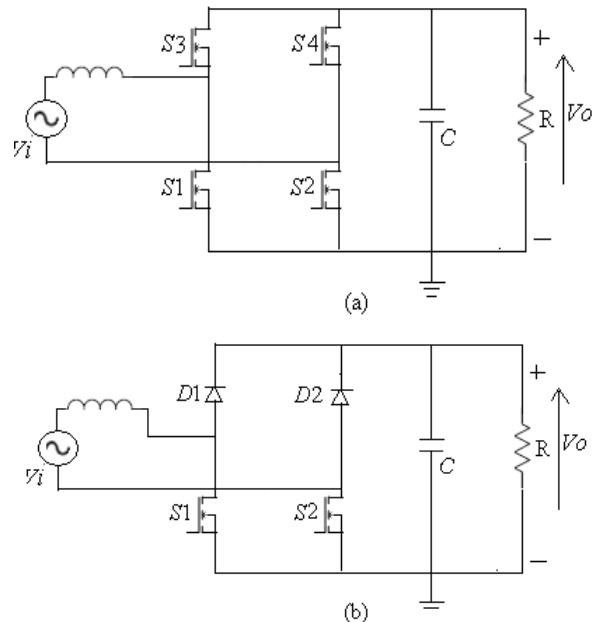

Fig. 6 H-bridge ac-dc boost converter (a) 4-switch, (b) 2-switch

To achieve the boost operation, the lower switches $\left(\mathrm{S}_{1}\right.$ and $\left.\mathrm{S}_{2}\right)$ these two converters should be able to conduct in both the directions. The bidirectional conduction capability of the two MOSFETs $\left(\mathrm{S}_{1}\right.$ and $\left.\mathrm{S}_{2}\right)$ can be used to achieve the boost operation. During the positive half cycle of the input voltage, $\mathrm{S}_{2}$ is kept on for the entire half cycle and the gate pulse to $S_{1}$ is controlled to achieve the boost operation. Likewise, in the negative half cycle, $S_{1}$ is kept on for the entire half cycle and $S_{2}$ is controlled. To achieve the boost operation, these two topologies use single inductor compared to the two inductors used in the proposed converter. However, there are several disadvantages in these Two H- Bridge converters. In H-bridge converters, there are two devices in the conduction path during charge or discharge of the boost inductor. But in the proposed converter, only a single device conducts during the charge or discharge of the inductors. In H-bridge converters, the MOSFETs, used for the boost operation $\left(\mathrm{S}_{1}\right.$ and $\left.\mathrm{S}_{2}\right)$, are operated for the entire cycle of the input ac voltage. But in the proposed converter, any MOSFET is operated only for a half cycle of the input ac voltage. Therefore, the device conduction losses in the proposed converter are reduced by more than a factor of two. The input voltage polarity has to be sensed to control $S_{1}$ and $S_{2}$, but in the H-bridge topologies, the input voltage source is floating with respect to the output voltage ground. Therefore, the implementation of the control circuit is difficult.

In the energy harvesting applications, as the power level is very low, these losses are significant compared to the total output power. As, the MOSFETs are designed for forward conduction, in the reverse conduction mode they offer higher on-state resistance. This further increases the conduction losses in the $\mathrm{H}$ bridge topology. It can be mentioned that although the proposed converter uses two inductors ( $L_{1}$ and $\left.L_{2}\right)$, they do not operate in the same half cycle. Therefore, their total losses are almost equal to the losses of the single inductor used in the H-bridge converters.

The rest of the paper organized in the following manner: Detailed analysis of the converter and the proposed control schemes to control the converter are explained in II. Design guidelines are explained in III. Proposed self-staring circuit and the converter control circuit implementation are explained in IV \& V. Simulations are presented in VI. Finally the conclusion is presented. 


\section{Direct Ac-To-Dc Converter}

The proposed direct ac-to-dc converter, 'as shown in Fig 5', consists of a buck-boost and a boost converter. The dc bus of the proposed converter is realized by using only one capacitor. The output capacitor $\mathrm{C}$ of this converter is charged by the boost converter (comprising inductor $L 1$, switch $S 1$, and diode, D1) and a buck- boost converter (inductor $L 2$, switch $S 2$, and diode $D 2$ ) during the positive half cycles and negative half cycles of the sinusoidal ac input voltage (vi), respectively. N-channel MOSFETs are utilized to realize the switches S1 and S2. It can be noted that the MOSFET s are subjected to reverse voltage by the ac output of the micro-generator. To block the reverse conduction, the forward voltage drop of the body diodes of the MOSFETs is chosen to be higher than the peak of the input ac voltage. Two schottky diodes (D1 and D2) with low forward voltage drop are used in the boost and the buck-boost converter circuits for low losses in the diodes. It can be noted that the diodes can be replaced by MOSFETs to further improve the efficiency of the converter.

Since the input current of a buck-boost converter is discontinuous. Hence, in this study, both the boost and buck-boost converter are operated in discontinuous mode (DCM) to control the output current of microgenerator. It can be noted that under constant duty cycle DCM operation, the input current is proportional to the input voltage at every switching cycle; therefore, the overall input current will be in-phase with micro-generator output voltage. A converter operating with a constant duty cycle has only fundamental and switching harmonic frequency components (much higher than the fundamental) thus, offers a resistive load to the micro-generator. By changing the equivalent load resistance offered to the micro-generator, the damping force in a microgenerator can be set to an optimal value to achieve maximum energy harvesting.

The proposed converter operates mainly in four modes during one cycle of the input ac voltage. The operating modes are discussed. The paths of the inductor currents, $i_{L 1}$ and $i_{L 2}$, in these modes, are depicted in thin lines. Mode1 and Mode 2 are for positive input voltage, when only the boost converter operates. Mode 3 and Mode 4 are for negative input voltage, when only buck-boost converter operates.

Mode 1 operation: This mode starts when the gate drive voltage, $\mathrm{Vg} 1$, is high, and hence, the boost-switch $\mathrm{S} 1$, is turned on. The current in the boost-inductor, L1, starts to build from zero. During this mode, gate drive voltage, $\mathrm{Vg} 2$, is low, so S2, is in 'off' condition and the diodes, D1 and D2 are also 'off'. The current direction during this mode 'as shown in Fig.7'.

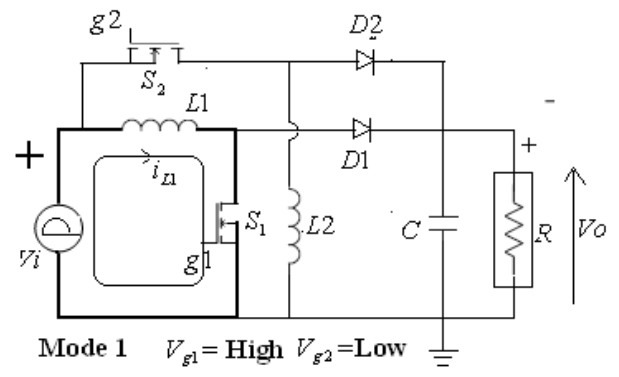

Fig .7 circuit of the converter during mode 1

Mode 2 operation: This mode starts at the end of Mode 1 when $\mathrm{Vg} 1$ becomes low and S1 turns off. During this mode, L1 forward biases D1 and its stored energy charges the output capacitor, C.S2 and D2 remains 'off' in this mode. It can be noted that during Mode 1 and Mode 2, the input applies reverse voltage across S2 but the higher forward voltage of the MOSFET body diode holds back any current conduction though it, the current directions during this mode 'as shown in Fig.8'.

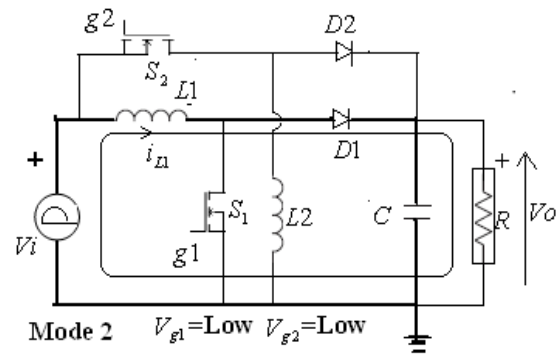

Fig .8 circuit of the converter during mode 2

Mode 3 operation: In this mode the gate drive voltage, Vg2, becomes high and S2 turns on. The current in the inductor L2 builds in the negative direction 'as shown in Fig.9'.. 


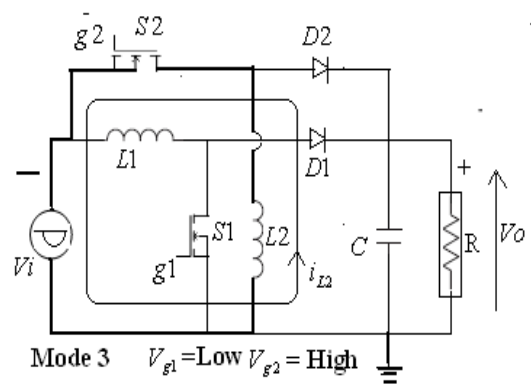

Fig.9 Circuit of the converter during mode 3

Mode 4 operation: The MOSFET S2 is turned off in this mode and the inductor L2 forward biases the diode D2. The energy stored in L2 charges the output capacitor $\mathrm{C}$, the current directions during this mode 'as shown in Fig.10'.

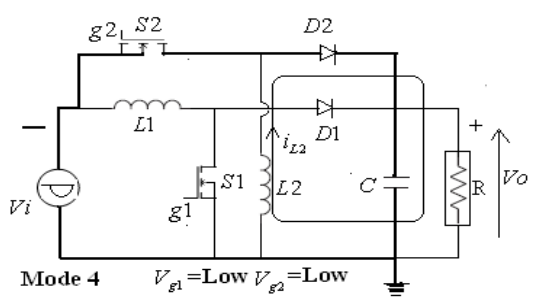

Fig .10 Circuit of the converter during mode 4

It should be noted that other than this modes there will be DCM operation of both the converters; in which all the switches and diodes will be in off conduction. It can be noted that the output capacitor $\mathrm{c}$ is charged in the same direction in each half-cycle. To achieve maximum energy harvesting by offering optimal damping to the micro-generators, the input current should be controlled. Hence, in this study, the boost and the buck-boost converters are controlled with fixed duty cycle to achieve an input current proportional to the input voltage.

\section{A. CONVERTER ANALYSIS}

Consider the input current waveform of the converter for the analysis is 'as shown in Fig.11 (a)'.

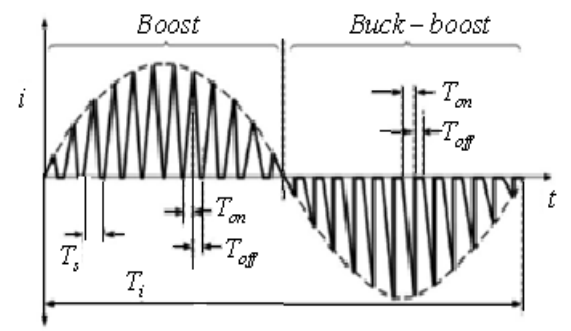

(a)

Consider any $k^{\text {th }}$ switching cycle of the boost converter 'as shown in Fig .11 (b)',
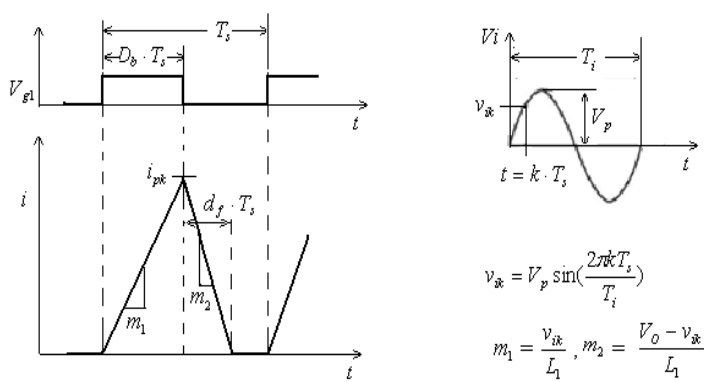

(b)

Fig .11 boost converter (a) Input currents and (b) gate drive signals and input voltage during a switching cycle.

Where $T s$ is the time period of the switching cycle, $D b$ is the duty cycle of the boost converter, $d_{f} T_{s}$ is the boost inductor current fall time (or the diode $D 1$ conduction time), $D c$ is the duty cycle of the 
buck- boost converter, $v_{i}$ is the input voltage of the generator with amplitude $V_{p}$, and $V_{o}$ is the converter output voltage.

Assuming the switching time period $\left(T_{s}\right)$ of the converter is much smaller than the time period of the input ac cycle $\left(T_{i}\right)$, during the boost converter operation, the input current $i$ and the boost inductor current $\left(i_{L 1}\right.$ ) are equal.

When the boost switch S1 is turned on, the current in the boost inductor L1 starts to build from zero and reaches a peak value.

The peak value of the inductor current $\left(i_{p k}\right)$ in the boost converter is
$i_{p k}=m_{1} D_{b} T_{s} \quad=\frac{v_{i k} D_{b} T_{s}}{L_{1}}$
(1) Where $m_{1}=\frac{v_{i k}}{L_{1}}$ and
$v_{i k}=V_{p} \sin \left(\frac{2 \pi k T_{s}}{T_{i}}\right)$

After the boost converter switch $S_{1}$ is turned OFF, the current in the inductor starts to fall until the switch $S_{2}$ is turned ON again in the next switching cycle from Fig .11(b).

The slope $\left(m_{2}\right)$ of this current is decided by the voltage across the inductor, in a $k^{\text {th }}$ switching cycle, the voltage across the inductor during the inductor current fall time is $V_{o}-v_{i k}$.

Therefore the inductor current fall time is

$$
d_{f} T_{s}=\frac{i_{p k}}{m_{2}}=\frac{i_{p k} L_{1}}{V_{O}-v_{i k}}
$$

Where $m_{2}=$ voltage across the inductor during the inductor $i$ fall time $t_{i} / L_{1}$

During the boost converter switch $S_{1}$ is OFF, the inductor current falls, and the energy stored in the inductor $\mathrm{L}$ is transferred to the load. In the boost converter, the energy to the output is more than energy stored in the inductor hence, for the equal duty cycles, input voltages, inductor values $\left(L_{1}=L_{2}\right)$,

Energy transferred during switch $\left(S_{1}\right)$ is turn on,

$$
E=V \cdot I \cdot t_{o n}=v_{i k} \cdot i_{p k} \cdot\left(D_{b} T_{s}\right)
$$

Energy transferred to the load during switch $\left(S_{1}\right)$ is turn off,

$$
E=V \cdot I \cdot t_{o f f}=\left(v_{i k}\right) \cdot i_{p k} \cdot\left(d_{f} T_{s}\right)
$$

During the $k^{\text {th }}$ switching cycle, the total energy $\left(E_{k b}\right)$ transferred from the input of the boost converter can be

$$
\begin{aligned}
E_{k b}=\frac{E_{\text {Ton }}+E_{\text {Toff }}}{2} & \frac{v_{i k} i_{p k} D_{b} T_{s}+v_{i k} i_{p k} d_{f} T_{s}}{2}=\frac{v_{i k} i_{p k}\left(D_{b}+d_{f}\right) T_{s}}{2}
\end{aligned}
$$

(6)

The averaged power supplied by the generator in the boost switching cycle is

$$
P_{k b}=\frac{E_{k}}{T_{s}}=\frac{v_{i k} i_{p k}\left(D_{b}+d_{f}\right)}{2}
$$

The number of switching cycles during the time period of one input ac cycle is defined as $N=T_{i} / T_{s}$ since the boost converter is operated for the half time period of tha input ac cycle $\left(\mathrm{T}_{\mathrm{i}} / 2\right)$.

The average input power $\left(P_{i b}\right)$ of the boost converter over this half cycle time period is

$$
P_{i b}=\left(\frac{2}{N}\right) \sum_{K=1}^{N / 2} P_{k b}=\left(\frac{2}{N}\right) \sum_{k=1}^{N / 2} \frac{v_{i k} i_{p k}\left(D_{b}+d_{f}\right)}{2}
$$


The average power supplied $\left(P_{k b}\right)$ can be

$$
\begin{aligned}
& P_{k b}=\frac{D_{b}^{2} T_{s}}{2 L_{1}} \cdot V_{p}^{2} \sin ^{2}\left(\frac{2 \pi}{T_{i}} t\right) \times \\
& V_{O}\left(V_{O}-V_{p} \sin \left(\frac{2 \pi}{T_{i}} t\right)\right)^{-1}
\end{aligned}
$$

The average input power of the boost converter $P_{i b}$ can be calculated by integrating the term in the summation over the half cycle $\left(T_{i} / 2\right)$ period of the input ac voltage.

$$
\begin{aligned}
& P_{i b}=\frac{2}{T_{i}} \int_{0}^{T_{I} / 2} \frac{D_{b}^{2} T_{s}}{2 L_{1}} \cdot V_{p}^{2} \sin ^{2}\left(\frac{2 \pi}{T_{i}} t\right) \times \\
& \frac{V_{O}}{V_{O}-V_{p} \sin \left(\frac{2 \pi}{T_{i}} t\right)} d t \\
& P_{i b}=\frac{D_{b}^{2} T_{S} V_{p}^{2}}{4 L_{1}} \cdot \beta
\end{aligned}
$$

Where $\beta=\frac{2}{\pi} \int_{0}^{\pi} \frac{1}{1-\left(\frac{V_{p}}{V_{O}}\right) \sin \theta} d \theta$

It can be noted that in (10), $\beta$ is constant for fixed values of $V p$ and $V o$. Also, it is noticed that for large switching frequency of the converter, the average power is independent of the micro generator output voltage.

In steady state, the average input power of the converter is equal to the sum of the average output power and the various converter losses. Hence, by defining the converter efficiency as $\eta$ for a load resistance $R$, the input power and the output power can be balanced as

$$
\frac{V_{p}^{2} D 2_{b}^{2} T_{s}}{4 L_{1}} \beta=\frac{V_{O}^{2}}{R} \frac{1}{\eta}
$$

From above, the duty cycle of the boost convereter $\left(D_{b}\right)$ can be obtained as

$$
D_{b}=\frac{2 V_{O}}{V_{p}} \sqrt{\frac{L_{1}}{R T_{s} \eta}} \frac{1}{\beta}
$$

Further, consider the operation of the buck-boost converter; in this case the input power is supplied only during the ON period of the switch $S 2$ (see Fig. 5). During the OFF period of the switch $S 2$, the input current is zero [see Fig. 11(a)].

Consider any $k^{\text {th }}$ switching cycle of the buck-boost converter 'as shown in Fig .10'. 

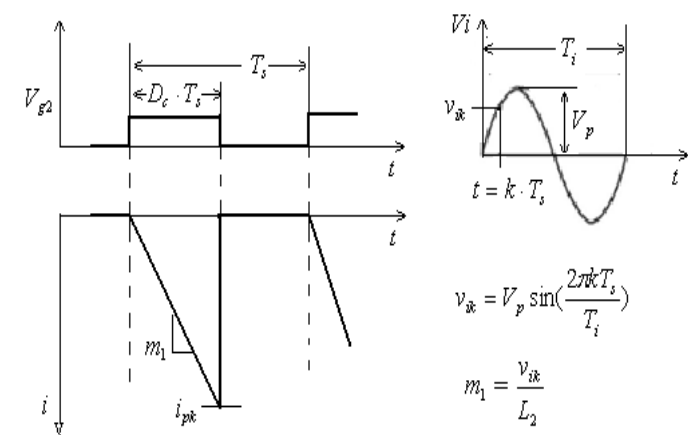

Fig. 12 buck-boost converter Input currents, gate drive signals and input voltage during a switching cycle.

Where $T s$ is the time period of the switching cycle, Dc is the duty cycle of the buck- boost converter, $V i$ is the input voltage of the generator with amplitude $V p$, and Vo is the converter output voltage.

The main application of a step-down/step-up or buck-boost converter is in regulated dc power supplies, where a negative-polarity output may be desired with respect to the common terminal of the input voltage, and the output voltage can be either higher or lower than the input voltage.

When the buck-boost switch $S_{2}$ is turned ON, the input provides energy to the inductor i.e. current flows through the inductor $L_{2}$, starts to build from zero and reaches a peak value.

The peak value of the inductor current $\left(i_{p k}\right)$ in the buck-boost converter is

$i_{p k}=m_{1} D_{c} \cdot T_{s}=\frac{v_{i k} D_{c} \cdot T_{s}}{L_{1}}$

When the buck-boost switch $S_{2}$ is turned OFF, the energy stored in the inductor is transferred to the output. No energy i.e. current is supplied by the input during this interval nothing but input current is zero. Hence for any $k^{\text {th }}$ switching cycle, the average power supplied by the buck-boost converter $P_{k c}$ is

$P_{k c}=\frac{v_{i k} i_{p k} D_{c}}{2}$

The average power can be expressed in integration form over a half cycle $\left(T_{i} / 2\right)$ period of the input ac voltage as

$$
\begin{aligned}
& P_{i c}=\frac{2}{T_{i}} \int_{0}^{T_{I} / 2} \frac{D_{c}^{2} T_{s}}{2 L_{2}} V_{p}^{2} \sin ^{2}\left(\frac{2 \pi}{T_{i}} t\right) d t \\
& P_{i c}=\frac{D_{c}^{2} T_{s} V_{p}^{2}}{4 L_{2}}
\end{aligned}
$$

By defining the converter efficiency as $\eta$ for a load resistance $R$, the input power and the output power can be balanced as in equation (15)

$\frac{V_{p}^{2} D_{c}^{2} T_{s}}{4 L_{2}}=\frac{V_{O}^{2}}{R} \frac{1}{\eta}$

From the above, the duty cycle of the buck boost converter $\left(D_{c}\right)$ can be obtained as

$$
D_{c}=\frac{2 V_{O}}{V_{p}} \sqrt{\frac{L_{2}}{R T_{s} \eta}}
$$




\section{B.CONVERTER CONTROL SCHEME}

Using equations (11) and (16), the duty cycle of the boost converter $D_{b}$ and duty cycle of the buckboost converter $D_{c}$ can be related as

$$
\frac{D_{b}}{D_{c}}=\sqrt{\frac{L_{1}}{L_{2}}} \frac{1}{\beta}
$$

Based on the equation (17), two different control schemes are proposed, for the boost and buck-boostbased converter to deliver equal average input power.

In scheme 1 , the values of the inductors are kept to be equal $(L 2=L 1)$ and the converters are controlled with different duty cycles such that it satisfies the condition:

$$
D_{C}=D_{b} \sqrt{\beta}
$$

In scheme 2 , both the boost and the buck-boost converters are controlled with same duty cycle ( $D_{b}=D_{c}$ ), whereas the inductor values are chosen to satisfy the condition:

$L_{1}=\beta L_{2}$ Fig.13'.

The variable $\beta$ from equation (10) is plotted as a function of the step-up ratio $(V o / V p)$ 'as shown in

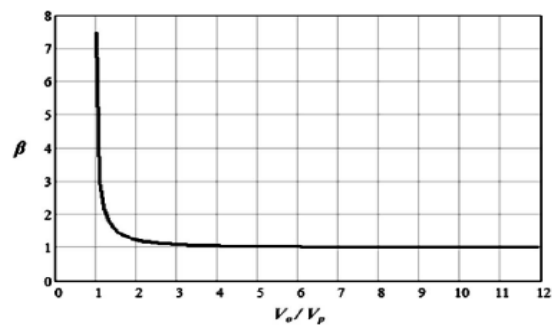

Fig. 13 plot for $V o / V p$ versus $\beta$

For large values of voltage step-up ratio $(V o / V p)$, the value of $\beta$ approaches to 1 . Hence, for higher voltage step-up ratio applications, the boost and the buck-boost converters can be designed with inductors of equal values and they can be controlled with the same duty ratio to successfully deliver the required average power to the output.

For $V o / V p \rightarrow 1$, the value of $\beta$ approaches to infinity. Therefore, from

Equation (10), the input power for the boost converter may seem to approach infinity as well. But in this case, the duty cycle of the boost converter $D_{b}$ approaches to zero for $V o / V p \rightarrow 1$. Therefore, no power is transferred from the input to the output and the equation remains valid even when $V o / V p=1$.

It can be mentioned that there could be two possible energy harvesting scenarios. One, in which the converter is controlled to harvest maximum power available from the vibrating body and the micro generator system and store it in an energy storage component (like battery) at the output. In this case, the output voltage is mainly decided by characteristics of the energy storage component. In the second scenario, the converter is controlled to harvest the amount of power demanded by the load while maintaining the desired output voltage. In this paper, the second scenario is considered to control the converter.

\section{Design Guidelines For Selection Of Converter}

The design steps for this converter are to select the MOSFETs, inductors, and the switching frequency of the converter. In a practical energy-harvesting scenario, the controller and the MOSFET driver circuit of the converter are required to be self starting and they should be powered by the energy harvesting system to power the controller and the drivers at the beginning, when the converter starts up.

For a given micro generator and a load, the input and output voltages are specified. Therefore, in this case, the voltage ratings of the MOSFETs are decided by the output voltage of the converter. The current rating of the MOSFETs has to be decided by the designer. It can be noted that the MOSFET carries maximum current at the peak of the input voltage from Fig.11 (a).

The maximum current Imax of the converter can be given as

$$
I_{\max }=\frac{V_{P} D}{L f_{s}}
$$


Here $f_{s}$ is the switching frequency of the converter, $L$ Is the inductance value for both boost and buck-boost converters, and $D$ Is the duty cycle of the converters $\left(D=D_{b}=D_{c}\right.$ ). From equations (14) and (20), the maximum current can be expressed as a function of average input power $P$ in as in equation (21).

$$
I_{\max }=\frac{4 P_{i n}}{V_{p} D}
$$

The relationship between the maximum input current and the duty cycle for different Input power is 'as shown in Fig. 14'.

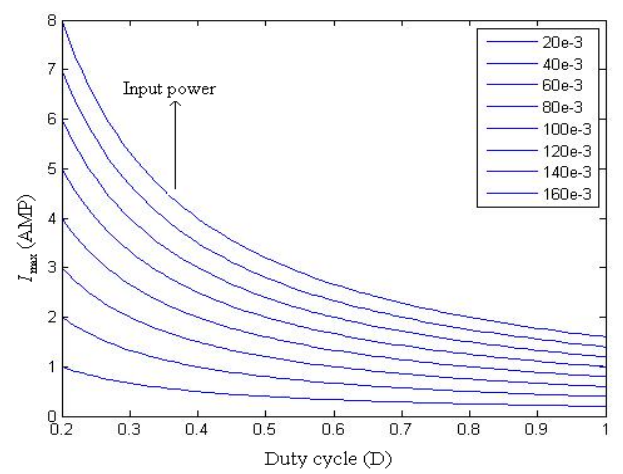

Fig.14 Duty cycle versus maximum input current

Using the (D V $\mathrm{I}_{\max }$ chart, the MOSFET current rating and converter duty cycle can be selected. With these selected values of the duty cycle $(D)$ and the MOSFET maximum current, the inductor value and the switching frequency can be obtained from equation $I_{\max }=\frac{V_{P} D}{L f_{S}}$

The relationship between the value of the inductor $(L)$ and the duty cycle over a range of switching frequency 'as shown in Fig.15'.

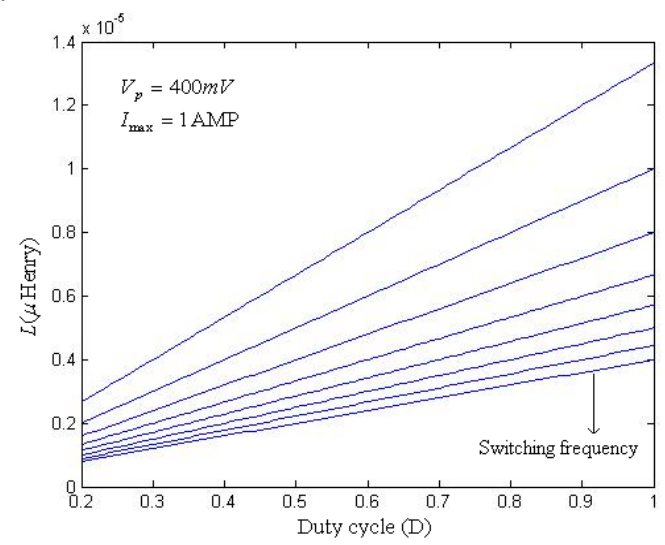

Fig. 15 duty cycle versus inductance

Using these charts and the equations, the initial values of the key components of the converter can be decided. It can be noted that the value of the maximum current $\left(I_{\max }\right)$ changes proportionally with the input power ( $P$ in) of the converter. Therefore, the size of the MOSFETs can be scaled as per the power requirement of the load. This is applicable for even low-power applications, demanding less than $1 \mathrm{mw}$ of power. The duty cycle and the frequency of the converter can be appropriately selected to choose the desired value of the inductor.

\section{Auxilliary Self-Starting Circuit}

The proposed self-starting auxiliary circuit utilizes a battery and schottky diodes for this purpose 'as shown in Fig.16'. 


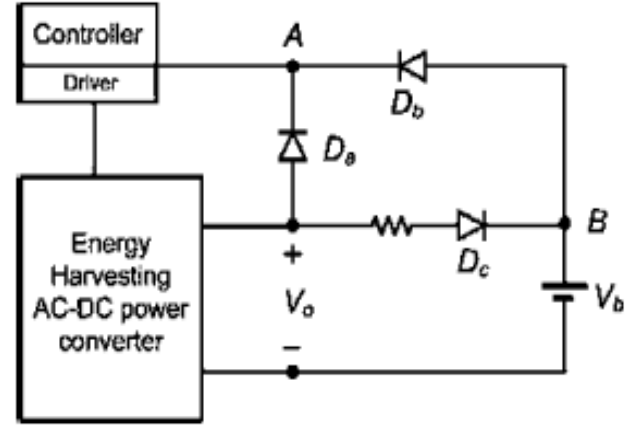

Fig. 16 Proposed auxiliary self-starting circuit using a battery

For the successful operation of this self-starting circuit, the battery nominal voltage $V_{b}$ should be less than the target output voltage $V_{O}$ minus the forward voltage drop of a diode $V_{f}$, and it should be above the minimum voltage requirements of the controller and driver circuits

$V_{b}<V_{O}-V_{f}$

Here we are considering, the target output voltage $V_{O}=3.3 \mathrm{~V}$ and forward voltage drop of the diodes $V_{f}=0.22 \mathrm{~V}$. A battery with nominal voltage $V_{b}=3 \mathrm{~V}$ is selected for the self-starting. At the beginning of the converter operation, when the output voltage $V_{O}$ is not available, the controller and the driver circuit will be powered by the battery through the Scotty diode $D_{b}$. This will allow the converter to charge the output capacitor to the reference voltage.

When the output voltage has reached the value $3.3 \mathrm{~V}$, the diode $D_{b}$ would be reversing biased; therefore, the battery will not be powering the controller and the driver. At this time, they will be powered from the converter output through the diode $D_{a}$. During this condition, the battery will remain under floating condition. It can be noted that only a very small amount of energy is taken from the battery for the startup of the energy harvesting system. Furthermore, the used energy of the battery can be replenished by recharging it from the output of converter through the diode $D c$.

Therefore, the entire amount of energy used for the converter operation, including the energy used during its starting, is harvested from the ambience. Therefore, with proper design and selection of the components, this auxiliary circuit can be used to operate the energy harvesting system virtually for indefinite period of time.

\section{Control Circuit Of The Converter}

The circuit diagram for the implementation of the proposed energy-harvesting converter and its control scheme 'as shown in Fig.17'.

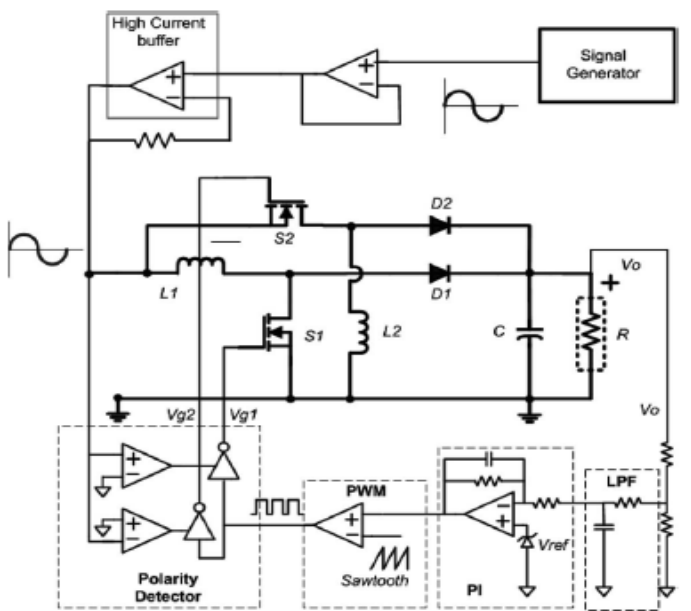

Fig. 17 circuit diagram of the energy-harvesting converter 
The resonance-based electromagnetic micro generator is modeled as an ac voltage source. A signal generator followed by a high-current buffer is used to realize the micro generator output voltage from Fig. 17 . This buffer can be realized by using a power operational amplifier in voltage follower mode.

The sensed output voltage of the converter is processed by a low-pass filter. The processed signal is compared with the reference voltage Vref. The error signal is used by the PI controller to estimate the control voltage that is compared with a saw-tooth waveform in the pulse width modulator (PWM). The pulse signal produced by the PWM is fed to the two buffers that can be enabled by external signals. The comparators in the polarity detector unit enable the appropriate buffers to produce the gate pulses ( $V g 1$ and $V g$ 2) that control the MOSFETs of the boost and buck-boost converter during appropriate half cycles

It can be summarized that the self-resistance and self-inductance of the electromagnetic micro generator are very small, therefore, the self-impedance of the micro generators are not included for the analysis of the proposed converter operation. However, it can be incorporated in the analysis, for the prediction of the performances of the entire energy harvesting system.

\section{Simulation Results}

A resonance-based electromagnetic micro-generator, producing $400 \mathrm{mV}$ peak sinusoidal output voltages, with $100-\mathrm{Hz}$ frequency is considered in this study for verification of the proposed converter topology. The reference output voltage $\left(V_{r e f}\right)$ is considered to be $3.3 \mathrm{~V}$. The energy-harvesting converter is designed for supplying power to a $200-\Omega$ load resistance, hence, supplying about $55 \mathrm{~mW}$ of output power.

The MOSFET is selected to realize the switches $S_{1}$ and $S_{2}$.the forward voltage of the selected MOSFET body diode is about $0.8 \mathrm{~V}$, which is higher than the peak input voltage. This prevents any reverse conduction in the MOSFETS. The nominal duty cycle of the converter is taken to be 0.7.The inductor is designed to have a standard value of $4.7 \mu H$ to realize $L_{1}$ and $L_{2}$. Based on these values the switching frequency is

$$
f_{s}=\frac{V_{P} D}{L I_{\max }}=\frac{0.4(0.7)}{4.7 * 10^{-6}}=50 \mathrm{kHZ}
$$

The diodes $D_{1} \& D_{2}$ are chosen to be schottky type with low forward voltage $0.23 \mathrm{~V}$.

The output capacitor value is $68 \mu \mathrm{F}$.Various values for circuit components of the designed converter are presented in Table I.

\begin{tabular}{|c|c|c|}
\hline Circuit Components & Name & Ratings \\
\hline Inductor & $L_{f}, L_{2}$ & $4.7 \mathrm{uH}$ \\
\hline Inductor resistance & $R_{i}$ & $30 \mathrm{~m} \Omega$ \\
\hline N - channel MOSFET & $S_{i} S_{2}$ & $20 \mathrm{~V}, 2 \mathrm{~A}$ \\
\hline MOSFET on state resistance & $R_{d s}$ em & $\begin{array}{c}150 \mathrm{~m} \Omega @ \\
V g s=3 \mathrm{~V}\end{array}$ \\
\hline Schottky Diode & $D_{l} \& D_{2}$ & $23 \mathrm{~V}, 1 \mathrm{~A}$ \\
\hline Schottky Diode forward voltage & $V_{f}$ & $0.23 \mathrm{~V}$ \\
\hline Load resistance & $R$ & $200 \Omega$ \\
\hline Capacitor & $C$ & $68 \mathrm{uF}$ \\
\hline Capacitor ESR & $R_{c}$ & $30 \mathrm{~m} \Omega$ \\
\hline
\end{tabular}

Table 6.1 Circuit components of the converter

To verify the proposed control schemes, at first simulation is carried out for the control scheme1, using $L_{2}=L_{1}=4.7 \mu H$ and duty ratio of the two converters are not equal. In the simulation duty cycle $D_{b}$ is estimated by a PI controller from Fig.7.

The buck-boost converter duty cycle $D_{c}$ is calculated from the estimated duty cycle $D_{b}$.

$$
\frac{D_{b}}{D_{c}}=\sqrt{\frac{L_{1}}{L_{2}}} \cdot \frac{1}{\beta} \quad\left(L_{2}=L_{1}=4.7 \mu H\right)=\sqrt{\frac{1}{\beta}}
$$




$$
\begin{aligned}
\beta & =\frac{2}{\pi} \int_{0}^{\pi} \frac{1}{1-\left(\frac{V_{p}}{V_{O}}\right) \sin \theta} d \theta \\
& =\frac{2}{\pi} \int_{0}^{\pi} \frac{1}{1-\left(\frac{0.4}{3.3}\right) \sin \theta} d \theta=1.0256 \\
& \frac{D_{b}}{D_{c}}=\sqrt{\frac{1}{\beta}}=\sqrt{\frac{1}{1.0256}}=0.9874 \\
D_{c}= & \frac{D_{b}}{0.9874}=\frac{0.7}{0.9874}=0.7089 \approx 0.71
\end{aligned}
$$

The input current of the boost converter and the input current of the buck-boost converter for load resistance $\mathrm{R}=200 \Omega$ 'as shown in Fig 18 and 19'.

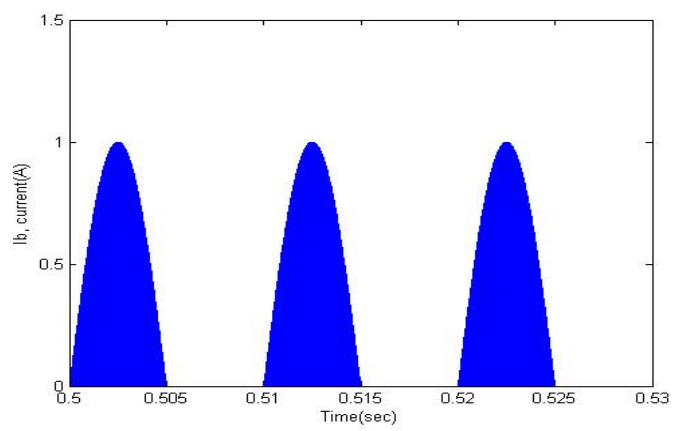

Fig 18 wave forms for input current of the boost converter

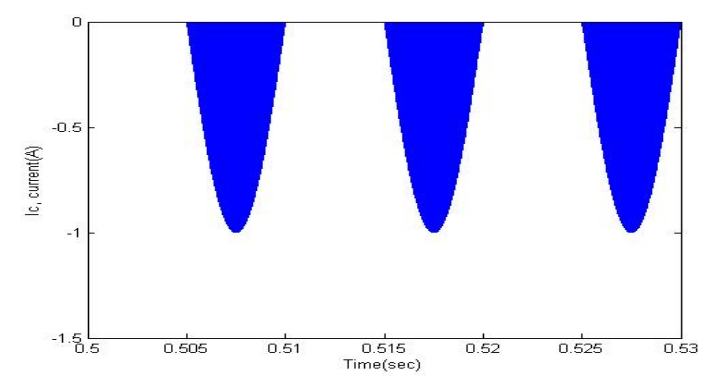

Fig. 19 wave forms for input current of the buck-boost converter

It can be seen that the boost converter is operated during the positive half cycle, while the buck-boost converter is operated during negative half-cycle of the micro generator output voltage. The converter output voltage and the duty cycles, estimated by the controller are 'as shown in Fig 20 (a), (b)'.

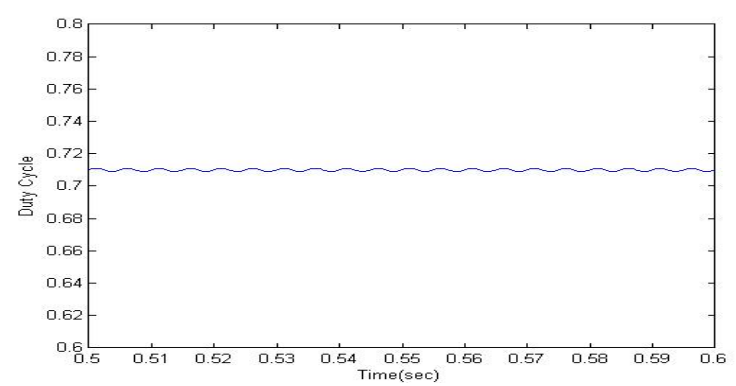

Fig .20 (a) equal duty cycles of the boost and buck-boost converters $\left(D=D_{b}=D_{c}\right)$ 


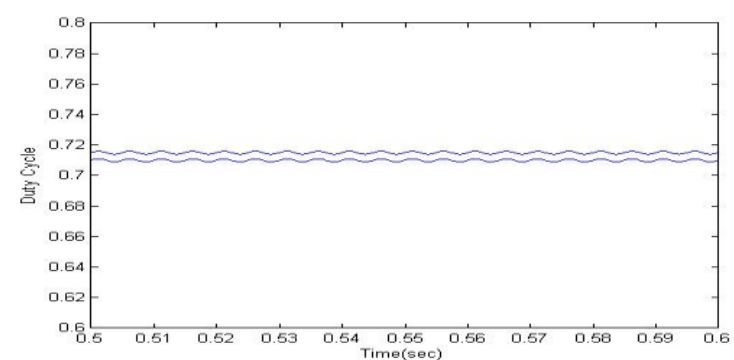

Fig .20 (b) unequal duty cycles of the boost and buck boost converters with $\left(L_{1}=L_{2}\right)$

The reference output voltage is considered as $3.3 \mathrm{~V}$ and the output voltage ripple is about $\pm 0.41 \mathrm{~V}$, which is $\pm 4.24 \%$ of the nominal output voltage. Duty cycle calculated from the solution is 'as shown in Fig 21 .

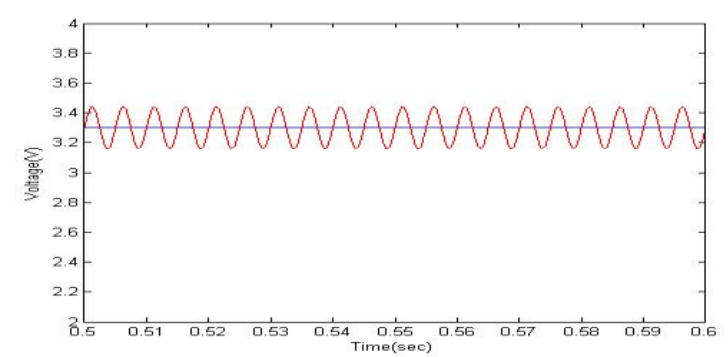

Fig .21 output voltage ripple with reference voltage

In this study, the output voltage to input voltage peak step-up ratio is

$$
\frac{V_{O}}{V_{p}}=\frac{3.3}{0.4}=8.25
$$

For high step-up ratio, the duty cycles of the converters with same inductor values will be almost equal. Hence, to achieve simple control structure, less component counts and for all other practical advantages, both the boost converter and the buck-boost converter can be controlled with same duty cycle for such high step-up applications. From figure 20, it can be seen that with the equal duty ratio control, the converter can successfully produce the desired output voltage.

To verify the operation of the converter under different load conditions, the load resistance is increased to $R_{L}=400 \Omega$

The input voltage, input current, and the estimated duty cycle by the controller are 'as shown in Fig 22'.

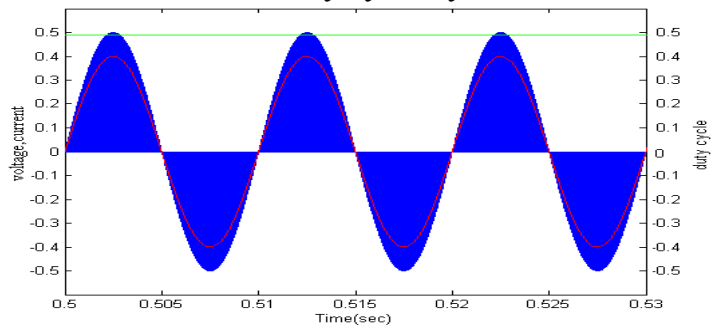

Fig.22 Input current, ac input voltage, and duty cycle $\left(D=D_{b}=D_{c}\right)$

For this load condition, the duty cycle value is about $D=0.49$. This matches with calculated duty cycle from $I_{\max }=\frac{V_{P} D}{L f_{s}}$

$D=\frac{I_{\max } L f_{s}}{V_{P}}=\frac{1(4.7 \mu) 50 \kappa}{0.4}=0.5$

The output voltage under this condition is 'as shown in Fig. 23'. 


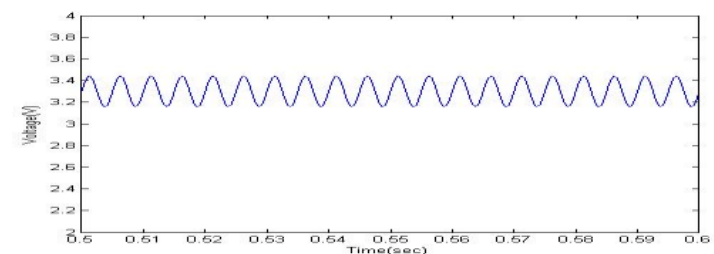

Fig 23 output voltage

The simulations are carried out with the self-starting circuit from Fig .16 proposed for the converter . The battery of the self-starting circuit is modeled as a constant voltage source of $3.0 \mathrm{~V}$. during start-up period of the converter, the power consumed by the control circuit from the battery and the converter is 'as shown in Fig 24 '.

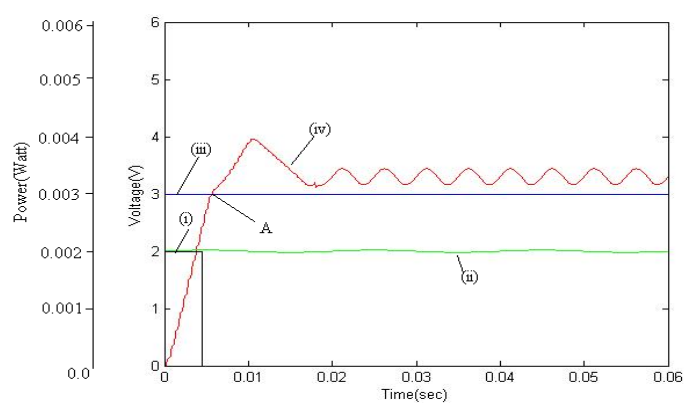

Fig.24. converter, battery power and voltages during self start-up. curves (i), (ii), (iii), and (iv) shows the power supplied by the battery, power draw from the converter output, battery voltage, and converter output voltage, respectively

The simulations are carried out for schemel i.e., with equal inductor values for high step-up ratio, the duty cycles of the converter with same inductor values will be almost equal. Hence, to achieve simple control structure, less component counts and for all other practical advantages, both the boost and buck-boost converter can be controlled with same duty cycle for such high step-up applications.

The simulations are carried out with the self-starting circuit proposed for the converter. It can be found that the start-up time taken by the converter is about $4.6 \mathrm{~ms}$. this is less than the half cycle period of the input ac voltage $(100 \mathrm{~Hz})$.

\section{Conclusion}

The presented direct ac-to-dc low voltage energy-harvesting converter avoids the conventional bridge rectification and achieves higher efficiency. The proposed converter consists of a boost converter in parallel with a buck-boost converter. The negative gain of the buck-boost converter is utilized to boost the voltage of the negative half cycle of the micro generator to positive ac voltage. Detailed analysis of the converter for direct act dc power conversion is carried out and the relations between various converter circuit parameters and control parameters are obtained. Based on the analysis, a simplified control scheme is proposed for high-voltage step-up application.

Design guidelines are presented for selecting values of the key components and control parameters of the converter. A self-startup circuit, using a battery only during the beginning of the converter operation, is proposed for the energy-harvesting converter operation and the implementation of the self-startup circuit and the control circuit of the converter is presented in details. Based on the analysis and the design guidelines, a prototype of the converter is developed. The proposed control scheme with the self-startup circuit is implemented and the converter is successfully operated to directly step-up the low ac voltage to a high dc voltage.

\section{References}

[1] J. A. Paradiso and T. Starner, "Energy scavenging for mobile and wireless electronics," IEEE Pervasive Comput., vol. 4, no. 1, pp. 18-27, Jan./Mar. 2005.

[2] S. Meninger, J. O. Mur-Miranda, R. Amirtharajah, A. P. Chandrakasan, and J. H. Lang, "Vibration-to-electric energy conversion," IEEE Trans. Very Large Scale Integr. Syst., vol. 9, no. 1, pp. 64-76, Feb. 2001.

[3] M. El-Hami, P. Glynne-Jones, N. M. White, M. Hill, S. Beeby, E. James, A. D. Brown, and J. N. Ross, "Design and fabrication of a new vibrationbased electromechanical power generator," Sens. Actuators A: Phys., vol. 92, pp. 335-342, 2001.

[4] T. M. Thul, S. Dwari, R. D. Lorenz, and L. Parsa, "Energy harvesting and efficient power generation from human activities," in Proc. Center Power Electron. Syst. (CPES) Semin., Apr. 2007, pp. 452-456.

[5] N. G. Stephen, "On energy harvesting from ambient vibration," J. Sound Vibrations, vol. 293, pp. 409-425, 2006. 
[6] J. R. Amirtharajah and A. P. Chandrakasan, "Self-powered signal processing using vibration-based power generation," IEEE $J$. Solid-State Circuits, vol. 33, no. 5, pp. 687-695, May 1998

[7] B. H. Stark, P. D. Mitcheson, M. Peng, T. C. Green, E. Yeatman, and A. S. Holmes, "Converter circuit design, semiconductor device selection and analysis of parasitics for micropower electrostatic generators," IEEE Trans. Power Electron., vol. 21, no. 1, pp. 27-37, Jan. 2006.

[8] C. B. Williams and R. B. Yates, "Analysis of a micro-electric generator for microsystems," in Proc. Int. Conf. Solid-State Sens. Actuators, 1995, pp. 369-372.

[9] P.D.Mitcheson, T. C. Green, E.M.Yeatman, and A. S. Holmes, "Architectures for vibration-driven micropower generators," J. Microelectromech. Syst., vol. 13, no. 3, pp. 429-440, Jun. 2004.

[10] S. Xu, K. D. T. Ngo, T. Nishida, G. B. Chung, and A. Sharma, "Low frequency pulsed resonant converter for energy harvesting," IEEE Trans. Power Electron., vol. 22, no. 1, pp. 63-68, Jan. 2007.

[11] S. Dwari, R. Dayal, and L. Parsa, "A novel direct AC/DC converter for efficient low voltage energy harvesting," in Proc. IEEE Ind. Electron. Soc. Annu. Conf., Nov. 2008, pp. 484-488.

[12] J. C. Salmon, "Circuit topologies for single-phase voltage-doubler boost rectifiers," IEEE Trans. Power Electron., vol. 8, no. 4, pp. 521-529, Oct. 1993

\section{BIOGRAPHY}

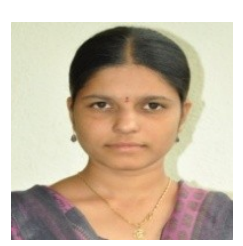

K Madhuri:Post graduate student in the department of Electrical and Electronics Engineering (P.E) at Sri Venkateshwara Engineering College, Suryapet, nalgonda, andrapradesh, India.

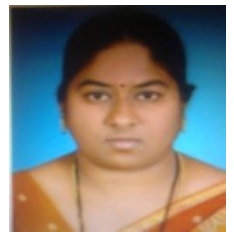

Dr.A.Srujana: B. Tech(Electrical), M.Tech (P.E), Ph.D (HVDC). Presently She is working as Professor \& H.O.D for Department of Electrical and Electronics Engineering at Sri venkateswara engineering college, Suryapet. 